\title{
PROTECTION OF LEP EXPERIMENTS AGAINST PARTICLE BACKGROUND AT HIGHEST BEAM ENERGIES
}

\author{
G. von Holtey, M. Lamont, CERN, Geneva, Switzerland
}

\begin{abstract}
The protection system used to shield LEP experiments against particle backgrounds has been upgraded in view of the much higher photon background rates expected at LEP2 energies. General experience with background rates in the LEP experiments at highest beam energies is reported and special features are highlighted.
\end{abstract}

\section{INTRODUCTION}

The main particle backgrounds at LEP are off-energy electrons and positrons produced by beam-gas bremsstrahlung and synchrotron radiation (SR) photons produced in the closest bending magnets and in quadrupoles in the straight sections on either side of the experimental detectors. While off-energy electron rates under normal vacuum conditions are acceptable and are not expected to increase with energy, the low energy SR photon background is many orders of magnitude larger than can be tolerated and increases rapidly with beam energy. The LEP collimator protection system, designed for $45 \mathrm{GeV}$, therefore had to be extended in order to cope with the much increased photon rates to be expected at twice the beam energy. The most important limit on photon background comes from aging of gas tracking detectors. They are designed to be sensitive to energy depositions of $\leq 1 \mathrm{keV}$ for minimum-ionizing charged particles and are therefore sensitive to $\mathrm{keV}$ photons, which interact in the gas or other local material [1].

After a description of the LEP2 background protection system, experience with detector backgrounds at high beam energies are reported.

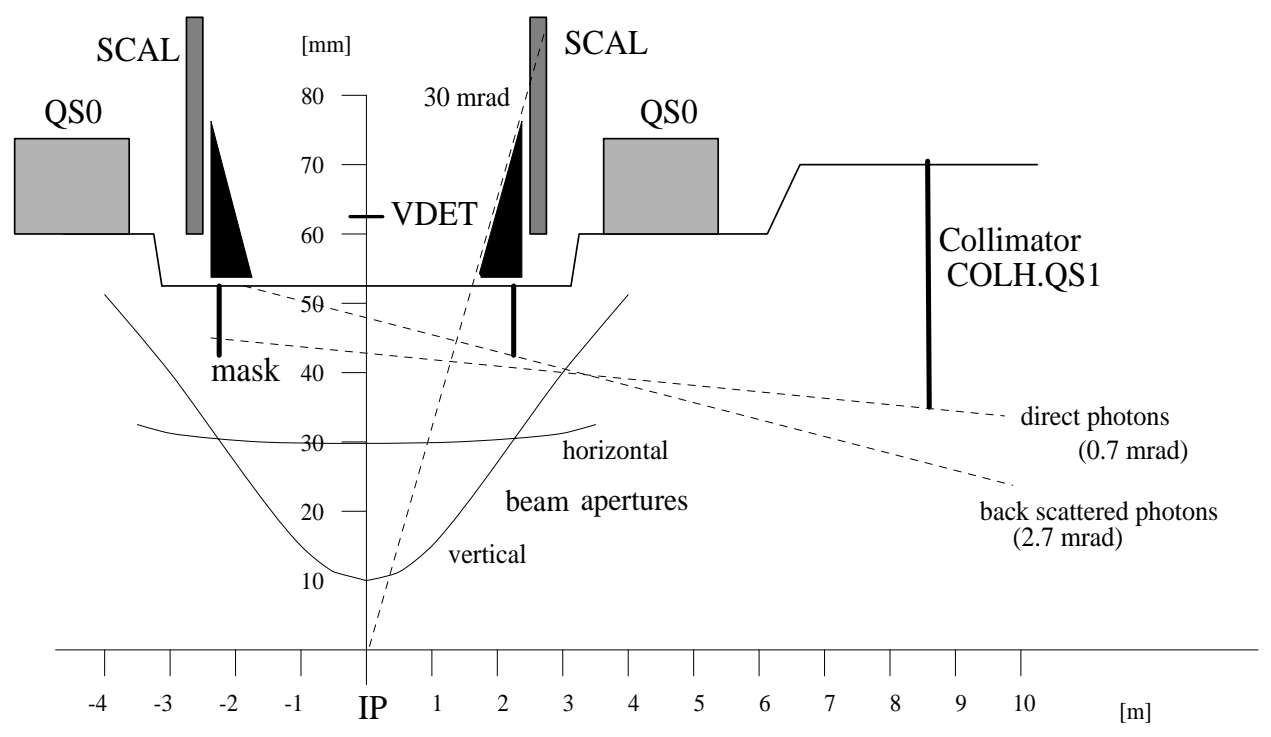

Figure 1: Schematic layout of SR-mask around a LEP IP, indicating the boundary conditions for a mask solution.

\section{LEP BACKGROUND PROTECTION}

The LEP detectors are protected against beam induced background particles by a system of movable two-jaw collimators placed at strategical locations in the experimental insertions. The LEP1 system is composed of 16 horizontal or vertical collimators for each experiment, plus six aperture limiting collimators located in a non-experimental insertion [2]. Simulations of SR-photon background at $90 \mathrm{GeV}$ indicated a large increase of the photon rate, mainly due to backscattering of photons from the first $50 \mathrm{~m}$ of vacuum chambers around the IP [3]. The solution proposed was to enlarge where ever possible vacuum chambers, to install eight more collimators per experiment, and as the main defense to incorporate cylindrical tungsten 
absorbers into the experimental vacuum chambers [4]. The functionality of these SR-masks and the MC predictions were verified with a test SR-mask installed in DELPHI during 1994 and 1995 using $45 \mathrm{GeV}$ and $65 \mathrm{GeV}$ beams [5]. A schematic layout of a SR-mask is shown in Figure 1, a comparison of measured background rates with different simulations is given in Figure 2.

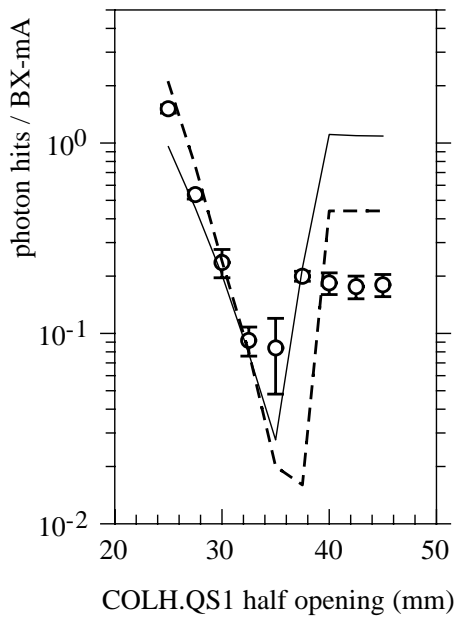

Figure 2: Monte Carlo predictions and measurements in the DELPHI TPC. Data points (photons per bunch crossing BX) are from the TPC, solid line MC [3], broken line MC [6]. $\mathrm{E}_{\text {beam }}=65 \mathrm{GeV}$.

Following the positive test results, the upgraded LEP2 protection system, including SR-masks, was installed for the 1997 run in all four experiments and has proven since to protect the detectors well at increasing beam energies up to the $94.5 \mathrm{GeV}$ run during 1998. As an example SR-photon and off-energy background rates for different energies and LEP optics measured by OPAL are shown in Figure 3. Rates increase linearly with current. The large fluctuation of the SR-photon rate reflects the strong dependence of this background on several machine parameters.

\section{LIMITATIONS OF THE MASK SOLUTION}

On the other hand, as the SR-masks are protruding into the vacuum chamber only $2.5 \mathrm{~m}$ away from the IP, they become very effective sources for low energy photons to be scattered into the detectors. The absorber rings are therefore shaped in such a way as to suppress all forward scattering. Back-scattering however from the downstream mask can not be avoided. The only solution is therefore to shield the downstream mask against direct photon hits.

This is achieved by a pair of additional horizontal collimators (COLH.QS3 or 4), placed close to a horizontal beam waist, some $50 \mathrm{~m}$ from the IP's. However, as LEP beam optics are considerably different in insertions IP4 and IP8 compared to the two other insertions, the horizontal beta-functions at the collimator location in IP4 and IP8 are two times larger and the mask protection is consequently not as good.

Sufficient protection can, however, still be achieved for both types of insertions as long as the density distribution of the horizontal beam tails stay close to Gaussian. An example is shown in Figure 4. Closing the protection collimators to $14 \sigma_{x}$, the minimum allowed setting before scraping into the beam, good protection is obtained in OPAL (IP6) and DELPHI (IP8). The measurement also demonstrates the limited margin left for the collimator opening in DELPHI, at which point the SR-mask is no longer protected and the photon rate starts to increase exponentially.

According to simulations for IP8 the downstream SRmasks are hit by about $3.10^{3}$ photons per bunch crossing (BX) with Gaussian beams of $3 \mathrm{~mA}$, against about $7.10^{6}$ photons/BX with exponential beam distributions. These photons are radiated by electrons on $\bullet 6$ sigma orbits in straight section quadrupoles 50 to $150 \mathrm{~m}$ upstream of the IP. The scattering probability from the tungsten mask into the detector for photons in the several $100 \mathrm{keV}$ energy range is of order $5.10^{-5}$, which amounts to several 100 photon hits/BX in the case of exponential beams. As the trip level for the central detectors is in the region of -10 photons/BX, such high rates could not be tolerated.
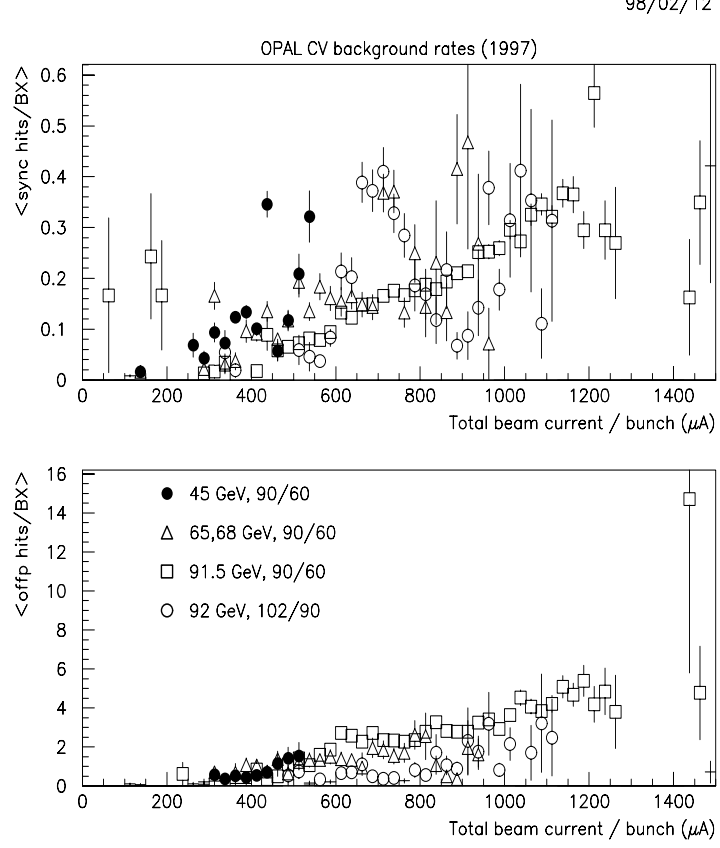

Figure 3: Hit rates per bunch crossing of synchrotron radiation photons and off-energy electrons measured in the OPAL Vertex Drift Chamber (CV) are shown as function of the total current in the two crossing bunches. The different symbols belong to different beam energies and LEP optics. One unit of 
'figure-of-merit' corresponds to a photon hit rate of about $0.6 / \mathrm{BX}$ and an electron hit rate of about 0.9/BX [7].

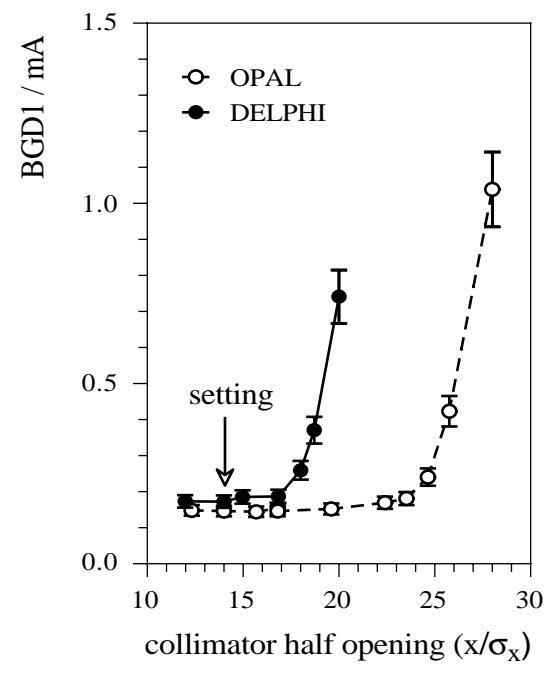

Figure 4: "Figure-of-merit" for SR-photons (BGD1) measured in DELPHI and OPAL as function of the opening of horizontal collimators COLH.QS3 and COLH.QS4, respectively. $\left(92 \mathrm{GeV}, \varepsilon_{\mathrm{x}}=30 \mathrm{~nm}\right)$.

While off-energy and SR-photon background rates stayed acceptably small under normal beam conditions during 1998 running at $94.5 \mathrm{GeV}$ with currents up to $3 \mathrm{~mA} /$ beam, occasionally large spikes and short periods of high photon background were observed, which led in many cases to detector trips, particularly in ALEPH (IP4) and DELPHI (IP8) [8].

These "photon storms" are believed to be due to resonance build-up of non-Gaussian beam tails in the horizontal plane. In the two insertions, where limited mask protection exists, SR-photons radiated by electrons in the far tails can reach the inner tip of the downstream mask and back-scatter into the detectors. Photon storms can occur several times during a fill, apparently uncorrelated to beam current. The slow exponential rise of the photon rate over several minutes can be stopped and restored by a very small increase of the horizontal tune of order 0.005 (see Figure 5).

In a MD experiment [9] photon storms could be triggered by reducing the horizontal tune to reach the $3 \mathrm{Qx}+\mathrm{Q} y=1$ resonance. No reduction of the beam lifetimes was observed, however, tail scans unveiled a strong increase of the particle density in the horizontal and vertical tails of one of the beams.

Further beam studies are needed to better understand the underlying effects during data taking conditions and to isolate the resonance's in question. Better collimator protection can be achieved if the horizontal aperture limit can be reduced below the present $12 \sigma_{x}$. Increasing the horizontal beta-value at the interaction points will reduce the beam size at the low-beta quadrupoles and allow for closer collimator settings.

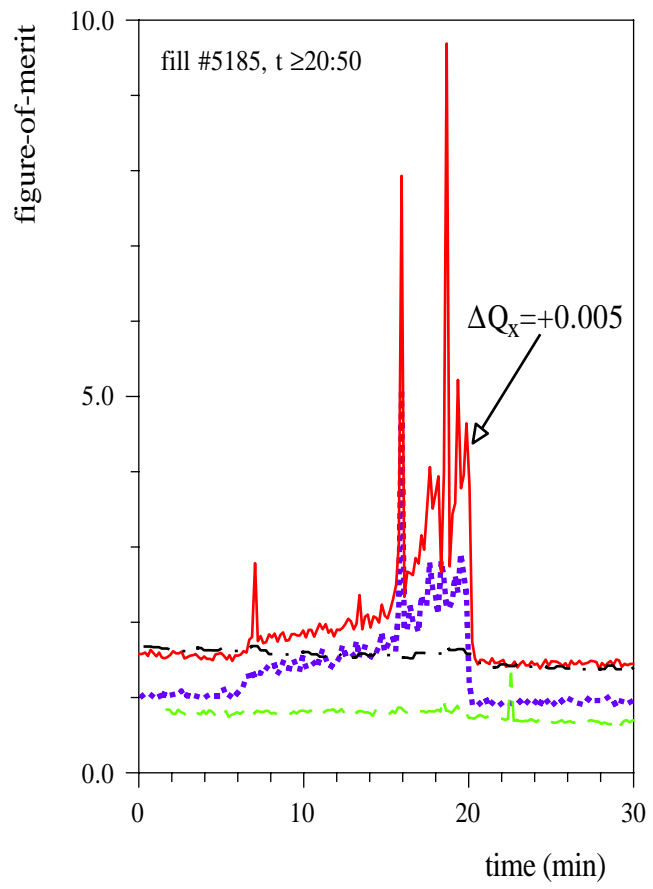

Figure 5: Background rates in ALEPH (dotted line), DELPHI (full line), OPAL (dash-dotted line) and L3 (dashed line) during a "photon storm" with data taking at $94.5 \mathrm{GeV}$. The exponential rise was stopped and the rates restored by $a Q_{x}$ trim of +0.005 .

\section{REFERENCES}

[1] J. Rothberg, Limitations due to background at LEP1, Proc. 5th Workshop on LEP Performance, Chamonix, 1995

[2] G. von Holtey and K.M. Potter, Estimation of synchrotron radiation background in LEP experiments, IEEE Particle Accelerator Conference, Washington. DC, 1987

[3] G. von Holtey, Simulations of photon backgrounds to LEP detectors at $90 \mathrm{GeV}$ beam energy, CERN SL/92-54, 1992

[4] G. von Holtey et al., Nucl. Instr. and Meth. in Phys. Res. A 403 (1998) 205

[5] The LEPC Working Group on LEP2 Backgrounds, CERN/LEPC93-10, 1993

[6] P. Roudeau, unpublished.

[7] P. Estabrookes, OPAL, private communication, 1998

[8] G. von Holtey, Particle backgrounds at the LEP detectors at $94.5 \mathrm{GeV}$, Proc. 9th Workshop on LEP Performance, Chamonix, 1999

[9] K. Affholderbach et al., "Beam tails and background storms with colliding beams at $94.5 \mathrm{GeV}$ ", SL-Note98-073 MD (1998) 